\title{
https://doi.org/10.1007/s00350-020-5563-6
}

\section{Solidarität und Verantwortung in der Corona-Krise}

\author{
Ad-hoc-Empfehlung \\ Berlin, 27.3.2020
}

\section{Deutscher Ethikrat}

\section{Einleitung}

Die gegenwärtige Pandemie fordert unsere Gesellschaft in beispielloser Form heraus. Zumindest aus der jüngeren Geschichte gibt es keine Erfahrungen mit entsprechenden gesundheitlichen Gefahren. Gleiches gilt für die aktuellen rigorosen, massiv und flächendeckend freiheitsbeschränkenden staatlichen Maßnahmen. Sie sollen dazu dienen, den exponentiellen Anstieg der Zahl infizierter und erkrankter Personen zu verhindern. Andernfalls könnte das Gesundheitssystem an seine Kapazitätsgrenzen gelangen. Bei rascher Zunahme schwerer Erkrankungsfälle könnte es $\mathrm{zu}$ einer Unterversorgung behandlungsbedürftiger Personen kommen - unabhängig davon, ob diese an der durch das neuartige Coronavirus verursachten Lungenerkrankung Covid-19 oder einer anderen Krankheit leiden. Allerdings haben die bereits ergriffenen Maßnahmen schon jetzt unvermeidliche Nebenfolgen für die wirtschaftliche und psychosoziale Lage, und bei besonders vulnerablen Personengruppen auch für deren gesundheitliche Situation.

Der ethische Kernkonflikt besteht in Folgendem: Ein dauerhaft hochwertiges, leistungsfähiges Gesundheitssystem muss gesichert und zugleich müssen schwerwiegende Nebenfolgen für Bevölkerung und Gesellschaft durch die Maßnahmen abgewendet oder gemildert werden. Garantiert bleiben muss ferner die Stabilität des Gesellschaftssystems. Hinzu kommt, dass noch unsicher ist, wann Impfstoffe, Medikamente, Therapien und Testverfahren zur Verfügung stehen werden, die eine nachhaltige Lösung ermöglichen.

Das erfordert eine gerechte Abwägung konkurrierender moralischer Güter, die auch Grundprinzipien von Solidarität und Verantwortung einbezieht. Eine besondere Spannung ergibt sich hierbei aus der unterschiedlichen primären Risikoverteilung: Einerseits ist nach heutigem Wissensstand bei vielen (vor allem Jüngeren) nur ein relativ milder Krankheitsverlauf zu erwarten; Kinder scheinen sogar kaum gefährdet. Andererseits besteht für bestimmte Risikogruppen (z. B. ältere Personen, Menschen mit Begleiterkrankungen bzw. chronisch Kranke) ein deutlich erhöhtes Mortalitätsrisiko.

Mit Blick auf die Spezifika des neuen Erregers, die Risikoverteilung und die zu erwartenden Belastungen des Gesundheits- und insbesondere des Krankenhaussystems erscheint eine Strategie des ,Laufenlassens“ unverantwortlich, die in der Hoffnung, die Epidemie werde zum Stillstand kommen, sobald genügend Personen die Infektion überstanden hätten (Gemeinschaftsschutz, auch „Herdenimmunität"), allein auf die rasche Verbreitung des Virus setzte. Anders zu beurteilen ist möglicherweise ein Vorgehen, das eine solche Strategie mit einem weitreichenden abschirmenden Schutz vulnerabler Gruppen verbindet. Aber

Deutscher Ethikrat,

Geschäftsstelle,

Jägerstraße 22/23, 10117 Berlin, Deutschland auch dabei ist zu bedenken, dass es gleichwohl zu einer Überlastung des Gesundheitssystems mit Gefahren für Leib und Leben aller kommen kann.

Aus ethischer Sicht sind - ungeachtet der hier nicht $\mathrm{zu}$ erörternden, durchaus strittigen Frage, ob hinreichende verfassungs- und einfachgesetzliche Grundlagen existieren - damit jedenfalls zur Zeit Freiheitsbeschränkungen vertretbar. Auch erheblich belastende Begleitschäden sind zumutbar. Je länger die Pandemie andauert, desto stärker sind allerdings nicht nur die unmittelbaren, sondern auch die vielfältigen, über den nationalen Kontext hinausweisenden Folgelasten sozialer und ökonomischer Art zu berücksichtigen.

\section{Zielsetzung der Ad-hoc-Empfehlung}

Vor diesem Hintergrund ist zunächst der epidemiologisch begründete Imperativ zu bekräftigen, dass die Ausbreitung des Virus jedenfalls erheblich verlangsamt, also die Infektionskurve abgeflacht werden muss (flatten the curve). Noch ist allerdings unklar, ob dies im angestrebten Maße gelingen und den erwünschten Effekt haben wird. Daher muss zugleich darüber nachgedacht werden, in welchem Ausmaß und wie lange die Gesellschaft erhebliche Einschränkungen ihres Alltagslebens verkraften kann. Zu klären ist, welche Maßnahmen in welchem Umfang und von welcher Dauer aus sozialer (rechtlicher, ökonomischer, politischer) und medizinischer Perspektive angemessen und auf längere Sicht vertretbar sind. Es geht um die Frage, wie die dann nahezu zwangsläufig entstehenden Normkollisionen und Konflikte aufzulösen oder wenigstens in ihren Folgen zu mildern sind. Hierzu will der Deutsche Ethikrat mit der vorliegenden Ad-hoc-Empfehlung einen Beitrag leisten. Sie konzentriert sich auf zwei wesentliche Aspekte:

Zum einen leistet sie ethische Orientierungshilfe in dramatischen Handlungs- und Entscheidungssituationen, vor allem solchen der sogenannten Triage. Hierzu werden basale Handlungsgebote und -verbote dargestellt. Zudem wird das Verhältnis dieser normativen Vorgaben zu konkreteren, in der ärztlichen Selbstregulierung entwickelten Regeln untersucht.

Zum anderen werden Kriterien und Verfahrensmaßgaben skizziert, wann und in welcher Weise von der gegenwärtig dominierenden Eskalations- auf eine Renormalisierungsstrategie zur Reduktion der Beschränkungen umgestellt werden kann. Renormalisierung bedeutet dabei nicht Rückkehr zur Normalität, ist also keineswegs mit einer Rückkehr zum status quo ante oder einer vollständigen Beseitigung der Gefahrensituation gleichzusetzen. Vielmehr geht es um die Frage, unter welchen Bedingungen von einem wieder akzeptablen Risikolevel als zwar notwendig unbestimmtem, aber gleichwohl gesellschaftlich nachvollziehbarem ,allgemeinen Lebensrisiko“ ausgegangen werden kann. Ein solches Vorgehen setzt sinnvollerweise auf klare zeitliche und sachliche Etappenziele, um gegenüber der jetzigen Beschränkungsstrategie Öffnungsperspektiven $\mathrm{zu}$ bieten. 
Insgesamt geht es in dieser Ad-hoc-Empfehlung darum, Politik und Gesellschaft dafür zu sensibilisieren, die dargelegten Konfliktszenarien auch als normative Probleme $\mathrm{zu}$ verstehen. Deshalb können und dürfen die anstehenden Entscheidungen nicht allein auf (natur-)wissenschaftlicher Basis erfolgen. Es wäre nicht nur eine Überforderung der Wissenschaft, wollte man von ihr eindeutige Handlungsanweisungen für das politische System verlangen. Es widerspräche auch dem Grundgedanken demokratischer Legitimation, würden politische Entscheidungen umfassend an die Wissenschaft delegiert. Wissenschaftliche Beratung der Politik ist wichtig, sie kann und darf diese aber nicht ersetzen. Denn wissenschaftliche Erkenntnisse geben keine hinreichende Auskunft über die Art und Weise ihrer Anwendung. Das ist eine gesamtgesellschaftliche Aufgabe, die im rechtlichen Rahmen von der demokratisch verantwortlichen Politik wahrzunehmen ist.

\section{Bewältigung dilemmatischer Entscheidungssituationen}

Möglicherweise lässt sich mittels der bereits beschlossenen Maßnahmen (Hygiene, körperliche Distanz etc.) die Anzahl der Neuinfektionen so weit reduzieren, dass insbesondere schwere Fälle, die intensivmedizinische Behandlung erfordern, für das Gesundheitssystem handhabbar bleiben. Das würde es ermöglichen, in nicht allzu ferner Zukunft die Strategie körperlicher Distanz stufenweise und kontrolliert abzubauen - zwar unter Inkaufnahme vermehrter Ansteckungen, damit aber auch mit einer Steigerung des Gemeinschaftsschutzes. Hierfür sollen die unten (unter 4.) angestellten Überlegungen Handreichung bieten.

Keineswegs fernliegend erscheint angesichts der Dynamik der Entwicklung allerdings das Alternativszenario, dass sich die vorhandenen und selbst potenziell weitere, noch eingriffsintensivere Maßnahmen auch dann als nicht ausreichend erweisen, wenn parallel die Kapazitäten der Intensivmedizin ausgebaut werden. Zwar werden in Deutschland gegenwärtig große Anstrengungen unternommen, diese Kapazitäten aufzustocken. Dennoch erscheint auch hierzulande eine Situation möglich, in der nicht mehr ausreichend intensivmedizinische Ressourcen für alle Patienten zur Verfügung stehen, die entsprechender Maßnahmen akut bedürfen. Dann könnte das ärztliche Personal gezwungen sein, ad hoc eine Triage vorzunehmen - also zu entscheiden, welche unter den Personen, die intensivmedizinische Behandlung und Versorgung benötigen, Priorität und damit etwa Beatmung oder eine extrakorporale Membranoxygenierung (ECMO) erhalten und wer nachrangig behandelt würde.

Das zwingt zur Auseinandersetzung mit den normativen Grundvorgaben, die für solche Bedingungen echter Knappheit in der (intensiv-)medizinischen Versorgung gelten. In einem zweiten Schritt sind diese Anforderungen mit den Vorgaben abzugleichen, die aktuell im Bereich der Medizin entwickelt werden.

\section{a) Grundvorgaben}

In Situationen, in denen der dringende Bedarf an lebenserhaltenden medizinischen Ressourcen nicht für alle gedeckt werden kann, die ihrer akut bedürfen, sind tragische Entscheidungen über Leben und Tod zu treffen. Für manche dieser Konstellationen gibt es keine rechtlich und ethisch umfassend befriedigende Lösung. Die Frage, wer aus welchen Gründen eine knappe Ressource erhält und wem sie verweigert wird, berührt zentrale Grundrechte der Betroffenen und wirft fundamentale Probleme der Verteilungsgerechtigkeit auf. Zur gegenwärtigen Krise gibt es bislang nur wenige konkrete Richtlinien für den Ernstfall. Die dazu jüngst in verschiedenen Ländern erarbeiteten Dokumente, darunter auch eine Stellungnahme sieben deutscher medizinischer Fachgesellschaften (siehe Link am Textende), gehen über theoretische Analysen hinaus und schlagen praktische Handlungskorridore, Priorisierungsalgorithmen und Entscheidungshilfen vor. In diesen spiegeln sich die angedeuteten ethischen und gerechtigkeitstheoretischen Probleme.

In Situationen katastrophaler Knappheit, wie sie für die gegenwärtige Lage auch in Deutschland nicht auszuschlieBen sind, erfährt die Grundorientierung ärztlichen Bemühens eine notwendige Erweiterung: von der ausschließlichen Konzentration auf das Wohl jedes einzelnen Patienten hin zur Berücksichtigung bestimmter Notwendigkeiten auch der öffentlichen Gesundheitsfürsorge unter den Bedingungen akuter Lebensgefahr für eine unabsehbar große Zahl weiterer, schwer und gleichartig Erkrankter. Diese Öffnung des ärztlichen Pflichtenkreises kann zu Kollisionen zwischen fundamentalen Maßgaben der Ethik und des Rechts führen. Im ernstesten Fall können sich diese als nahezu unlösbare Dilemmata erweisen.

Verbindlicher Rahmen auch für die ärztliche Ethik sind fundamentale Vorgaben der Verfassung: Die Garantie der Menschenwürde fordert eine egalitäre Basisgleichheit und statuiert damit einen entsprechenden basalen Diskriminierungsschutz aller. Für den Staat als unmittelbaren Adressaten der Grundrechte gilt darüber hinaus der Grundsatz der Lebenswertindifferenz: Be- oder gar Abwertungen des menschlichen Lebens sind ihm untersagt. Jede unmittelbare oder mittelbare staatliche Unterscheidung nach Wert oder Dauer des Lebens und jede damit verbundene staatliche Vorgabe zur ungleichen Zuteilung von Überlebenschancen und Sterbensrisiken in akuten Krisensituationen ist unzulässig. Jedes menschliche Leben genießt den gleichen Schutz. Damit sind nicht nur Differenzierungen etwa aufgrund des Geschlechts oder der ethnischen Herkunft untersagt. Auch eine Klassifizierung anhand des Alters, der sozialen Rolle und ihrer angenommenen „Wertigkeit" oder einer prognostizierten Lebensdauer muss seitens des Staates unterbleiben.

Diese Vorgaben widerstreiten einem rein utilitaristischen Modus des Abwägens im Sinne einer bloßen Maximierung von Menschenleben oder Lebensjahren. Stattdessen geht es um die Garantie fundamentaler Prinzipien des Rechts durch den Staat. Insoweit ist angesichts drohender Dilemmata in möglichen Triage-Situationen zunächst das normative Grundprinzip aller hierfür orientierenden Maßgaben festzuhalten: Selbstverständlich ist alles Zulässige zu unternehmen, um so viele Menschenleben wie möglich zu retten. Doch dürfen die dafür erforderlichen Maßnahmen den Rahmen verfassungsrechtlich zwingender Gebote nicht überschreiten. Auch persönliche ethische Überzeugungen, die etwa eine reine Ergebnisorientierung und mit ihr die unbedingte Maximierung der Zahl geretteter Menschenleben fordern mögen, können ein Handeln, das die skizzierten Grenzen des Verfassungsrechts überschritte, nicht rechtfertigen.

Der Staat darf menschliches Leben nicht bewerten, und deshalb auch nicht vorschreiben, welches Leben in einer Konfliktsituation vorrangig zu retten ist. Selbst in Ausnahmezeiten eines flächendeckenden und katastrophalen Notstands hat er nicht nur die Pflicht, möglichst viele Menschenleben zu retten, sondern auch und vor allem die Grundlagen der Rechtsordnung zu garantieren.

Die Möglichkeiten des Staates, abstrakt bindende Vorgaben für die Allokation knapper Ressourcen zu machen, sind somit begrenzt. Die grundrechtlichen Direktiven beschreiben im Wesentlichen negativ den Bereich des nicht mehr Zulässigen. Positive Orientierung für die konkrete Auswahlentscheidung in der Klinik bieten sie dagegen kaum. Das bedeutet nicht, dass keine handlungsleitenden Vorgaben konzipierbar wären. Aus dem Verbot einer eigenen staatlichen Bewertung folgt nicht, dass entsprechende Entscheidungen nicht akzeptiert werden können. Es ist 
daher sinnvoll, unterschiedliche Ebenen normativer Konkretisierung zu verbinden. Das verweist auf die Funktion zum Beispiel der Fachgesellschaften, die im Rahmen der vorgenannten Grundvorgaben wichtige Orientierungshilfen geben können und sollten, welche inhaltlich über das hinausgehen, was staatlicherseits zulässig wäre.

Die damit hervorgehobene Primärverantwortung der Medizin für einzelne Entscheidungen und deren Umsetzung folgt aus den erwähnten Grenzen des staatlich Normierbaren, die dem Verfassungsrecht geschuldet sind. Doch sollte diese Verantwortung keinesfalls nur ,am Krankenbett“, also im Rahmen der individuellen Arzt-Patienten-Beziehung, wahrgenommen werden. Schon aus Gründen der Gleichbehandlung, aber auch um der allgemeinen Akzeptanz willen bedarf es vielmehr weithin einheitlicher Handlungsmaximen für den klinischen Ernstfall. In der Reaktion auf die aktuelle Krise sind, wie angedeutet, erste Empfehlungen medizinischer Fachgesellschaften erschienen.

\section{b) Ethische Konflikte im Einzelfall}

In Notlagen, in denen weniger Beatmungsplätze vorhanden sind als akut gebraucht würden, sind zwei Grundszenarien zu unterscheiden:

- Triage bei Ex-ante-Konkurrenz: Damit sind Fälle bezeichnet, in denen die Zahl der unbesetzten Beatmungsplätze kleiner ist als die Zahl der Patienten, die ihrer akut bedürfen. Die hier unausweichlichen Entscheidungen sind normativ weniger problematisch, wenngleich auch sie für die entscheidenden Personen mit schweren seelischen Belastungen verbunden sind. Patienten, denen danach die Behandlung vorenthalten wird, werden von den medizinischen Entscheidern nicht etwa durch Unterlassen ,getötet", sondern aus Gründen einer tragischen Unmöglichkeit vor dem krankheitsbedingten Sterben nicht gerettet. Hier gilt der Grundsatz, dass niemand zu Unmöglichem verpflichtet sein kann. Das Recht bietet für diese Entscheidung keine positiven Auswahlkriterien. Sichergestellt werden muss jedoch, dass unfaire Einflüsse bei der Entscheidung nach aller Möglichkeit ausgeschlossen werden, etwa solche im Hinblick auf sozialen Status, Herkunft, Alter, Behinderung usw. Aus ethischer Sicht sollte die Entscheidung nach wohlüberlegten, begründeten, transparenten und möglichst einheitlich angewandten Kriterien geschehen.

- Triage bei Ex-post-Konkurrenz: In diesem Szenario, in dem alle verfügbaren Beatmungsplätze belegt sind, müsste - bei für alle Versorgten fortbestehender Indikation, deren Feststellung der ärztlichen Urteilskraft obliegt - die lebenserhaltende Behandlung eines $\mathrm{Pa}-$ tienten beendet werden, um mit dem dafür erforderlichen medizinischen Gerät das Leben eines anderen zu retten. Solche Entscheidungen sind erheblich problematischer. Hier können Grenzsituationen entstehen, die für das behandelnde Personal seelisch kaum $\mathrm{zu}$ bewältigen sind. Wer in einer solchen Lage eine Gewissensentscheidung trifft, die ethisch begründbar ist und transparenten - etwa von medizinischen Fachgesellschaften aufgestellten - Kriterien folgt, kann im Fall einer möglichen (straf-)rechtlichen Aufarbeitung des Geschehens mit einer entschuldigenden Nachsicht der Rechtsordnung rechnen. Objektiv rechtens ist das aktive Beenden einer laufenden, weiterhin indizierten Behandlung zum Zweck der Rettung eines Dritten jedoch nicht. Hier muss an den oben formulierten prinzipiellen Imperativ erinnert werden: Auch in Katastrophenzeiten hat der Staat die Fundamente der Rechtsordnung zu sichern. Weniger noch als selbst zahlreiche tragische Entscheidungen in Lebens- und Sterbensnotfällen könnten Staat und Gesellschaft eine Erosion dieser Fundamente ertragen.

\section{Legitimitätsvoraussetzungen und negative Auswirkungen des weitgehenden gesellschaftlichen Lockdowns}

Wie lange die teilweise gerade erst in Kraft gesetzten Maßnahmen zur Beschränkung der Pandemie aufrechterhalten bleiben sollen, ist derzeit weder entschieden noch entscheidbar. Nach jüngsten öffentlichen Auskünften von Virologen und Epidemiologen könnte die Pandemie in Abhängigkeit von der Verfügbarkeit erforderlicher Medikamente und Impfstoffe noch ein bis zwei Jahre dauern. Vor diesem Hintergrund stellt sich die Frage, wie lange die Maßnahmen zur zeitlichen Verzögerung des pandemischen Verlaufs und dabei insbesondere die zur Reduktion körperlicher Kontakte gerechtfertigt sind. Die Rechtfertigung dieser Maßnahmen des Lockdowns bedarf von deren Einführung an und für jeden Zeitpunkt ihrer Dauer einer überaus komplexen Güterabwägung unter den Bedingungen von Unsicherheit.

\section{a) Ausgangspunkt: Strategieentscheidungen \\ im ethischen Korridor von Solidarität und Verantwortung}

Der ethische Grundkonflikt erfordert die Abwägung des erhofften Nutzens einer Strategie körperlicher Distanz für die dauerhaft belastbare Aufrechterhaltung des Gesundheitssystems mit den befürchteten oder unmittelbaren Schäden für die politische, soziale, wirtschaftliche und kulturelle Lebenslage derjenigen Personen oder Personengruppen, die von dieser Strategie unmittelbar oder mittelbar betroffen sind. Solche Abwägungen, die immer auch Nützlichkeitserwägungen einschließen, sind ethisch einerseits unabdingbar, andererseits nur insofern zulässig, als sie keine Grund- und Menschenrechte oder weitere fundamentale Güter auf Dauer aushöhlen oder sogar zerstören. Auch der gebotene Schutz menschlichen Lebens gilt nicht absolut. Ihm dürfen nicht alle anderen Freiheits- und Partizipationsrechte sowie Wirtschafts-, Sozial- und Kulturrechte bedingungslos nach- bzw. untergeordnet werden. Ein allgemeines Lebensrisiko ist von jedem zu akzeptieren.

\section{aa) Solidaritätskonflikte}

Gerade in diesen Tagen der Krise zeigt sich, wie groß die Solidaritätsressourcen in unserem Lande sind. Solidarität bedeutet die Bereitschaft zu prosozialen Handlungen auf der Grundlage relevanter Gemeinsamkeit, die der solidarbereiten Person etwas abverlangen. Sie besteht weder automatisch noch unbegrenzt. Mag der Impuls zur solidarischen Hilfsbereitschaft am Anfang von jenem elementarmenschlichen Mitgefühl ausgehen, das nahezu jede Person angesichts schwerer Bedrohungen anderer empfindet, so muss solches Mitempfinden immer noch übersetzt werden in konkretes Handeln. Solidarität hängt von verschiedenen Faktoren ab: Es muss ein Grundgefühl von Zusammengehörigkeit oder wenigstens gemeinsamer Betroffenheit in einer Gefährdungssituation bestehen. Es müssen den Solidarbereiten ausreichende materielle oder immaterielle Mittel zur Verfügung stehen, ihren Wunsch zur Hilfe auch in die Tat umzusetzen. Die solidarisch handelnden Personen müssen unter Umständen sogar bereit sein, in zumutbaren Grenzen sich selbst zu gefährden. Dies geschieht allerdings regelmäBig in der Erwartung, Nutzen und Lasten würden jedenfalls auf lange Sicht fair und gerecht verteilt. Alle an solidarischen Praxisformen beteiligten Personen sollten sich fragen, welche Einbußen und Kosten man wem mit Gründen ansinnen darf - im aktuellen Fall also etwa, wem welche Einbußen in der politischen, sozialen, wirtschaftlichen oder kulturellen Lebensweise zugemutet werden dürfen.

\section{bb) Verantwortungszuweisung und Risikoakzeptanz}

Entsprechende Entscheidungen müssen zunächst von jeder einzelnen Person getroffen werden. In den Gestaltungsbe- 
reich dieser höchstpersönlichen Eigenverantwortung gehört auch das Bewusstsein, dass die eigenen Entscheidungen und die eigene Lebensführung immer Konsequenzen auf die Entscheidungen und die Lebensführung anderer zeitigen. Das gilt etwa für den Fall, dass Angehörige von Hochrisikogruppen für sich zu entscheiden geneigt sind, von besonderen Schutzstrategien zugunsten anderer Optionen abzusehen. Eigenverantwortung ist immer relational. In diesem Rahmen des Relationalen müssen auch Personen jener wachsenden Gruppe ihre Entscheidungen abwägen, die die Infektion und Erkrankung überstanden haben und nach derzeitigem Stand der Wissenschaft zumindest vorerst weder für andere ansteckend noch selbst re-infektionsgefährdet sind. Unabhängig davon, ob dies staatlicherseits verordnet werden kann, ist es Ausdruck solidarischer Eigenverantwortung derjenigen, die nach einer Infektion immun sind, die wiedererlangte Freiheit und Handlungsmöglichkeit im Sinne der Solidargemeinschaft auch für die Überwindung dieser schweren Krise einzusetzen. Dies birgt zugleich die Chance, das gesellschaftliche Miteinander auch mit langfristiger Wirkung zu stärken.

Über solidarische Eigenverantwortung hinaus darf der Staat auch Risikofestlegungen und Verantwortungszuweisungen vornehmen, um die elementaren Funktionsbedingungen des gesellschaftlichen Lebens zu sichern. Dazu gehört die Einbindung unterschiedlicher Akteure (Einzelpersonen, Gruppen, Verbände des öffentlichen Lebens, staatliche Institutionen) im Rahmen einer Multiakteursverantwortung. In den Grenzen der Verfassung darf der Staat auch über das Maß des vom Einzelnen und der Gesellschaft hinzunehmenden Risikos entscheiden. Schon jetzt bedarf es einer breitangelegten öffentlichen Diskussion, um aus der gegenwärtigen Krise zu lernen und entsprechende Konsequenzen zu ziehen.

\section{b) Voraussetzungen und Folgen eines strukturierten Renormalisierungsprozesses}

Viele Stellungnahmen zum Thema konzentrieren sich derzeit auf die Frage der Legalität und moralischen Legitimität der aktuellen Maßnahmen. Diese berechtigte Frage nach dem „Wie weit?“ ist indes um die mittel- und langfristig bedeutsamere Frage zu ergänzen, unter welchen Voraussetzungen ein gesellschaftlich erörterter und möglichst breit konsentierter Wechsel vom Lockdown zur Wiederaufnahme des „Normalbetriebs“ erfolgen kann. Über das grundlegende Ziel hinaus, die Infektionsrate zu reduzieren, geht es schon jetzt darum zu überlegen, wie eine geordnete Rückkehr zu einem einigermaßen „normalen“ gesellschaftlichen und privaten Leben sowie zu regulären wirtschaftlichen Aktivitäten erfolgen kann, um die ökonomischen, kulturellen, politischen und psychosozialen Schäden möglichst gering zu halten.

Dabei kommt der Bereitschaft, die Maßnahmen des Lockdowns im Sinne einer gemeinschaftsstiftenden Solidarität zu akzeptieren, besondere Bedeutung zu. Sie ist im wesentlichen Umfang abhängig von zwei Faktoren:

- Der permanenten Kontrolle von Erforderlichkeit und Angemessenheit der ergriffenen Maßnahmen. Das Verhältnismäßigkeitsprinzip verlangt insoweit auch, und in der gegenwärtigen Situation im besonderen Maße, ein Einbeziehen der Zeitdimension.

- Damit eng verknüpft ist das Postulat, der Öffentlichkeit zu erläutern, wie und unter welchen Voraussetzungen Wege zurück in einen Zustand der „Normalität" beschritten werden können.

Beide Faktoren lassen sich zusammenführen im Begriff der Öffnungsperspektiven. In normativer Hinsicht wird damit zum Ausdruck gebracht, dass jede Grundrechtseinschränkung zu jedem Zeitpunkt rechtfertigungsbedürftig ist. Sozialpsychologisch ermöglicht die Öffnungsperspektive, dass die von ihr projizierte zeitliche Begrenzung die
Akzeptanz der Freiheitsbeschränkungen in der Ist-Situation erhöhen kann. Umgekehrt gilt: Ungewissheit über das Ende solcher Maßnahmen führt mit zunehmender Dauer zur Entsolidarisierung und Demotivation. $\mathrm{Zu}$ betonen ist, dass die genannten sozialpsychologischen Aspekte durchaus auch Einfluss haben auf die normative Bewertung der Angemessenheit bzw. Zumutbarkeit von Freiheitsbeschränkungen.

Systemgefährdungen durch die Beschränkungen lassen sich in nahezu allen gesellschaftlichen Teilsystemen prognostizieren: In der Wissenschaft etwa, wenn weder die Forschungsinfrastrukturen aufrecht erhalten werden können noch der fachliche Austausch im erforderlichen Maß gepflegt werden kann. Auch das Bildungssystem wird seiner gesamtgesellschaftlich bedeutsamen Funktion nicht mehr gerecht. Sport und Kultur sind erheblich beeinträchtigt. Von besonderer Bedeutung sind darüber hinaus:

- Sozialpsychologische Folgen: Der Lockdown zielt darauf ab, den Anstieg der Infektionen zu bremsen, um eine Überforderung der Gesundheitsversorgung zu verhindern. Zur Rettung des Lebens schwer Erkrankter ist dies notwendig. Aber auch erwartete Nebenwirkungen bedrohen die Gesundheit, möglicherweise sogar das Leben insbesondere solcher Personen, die vulnerablen Gruppen angehören. Dazu gehören:

- Patienten, deren medizinische Behandlung als derzeit nicht zwingend notwendig ausgesetzt wird,

- Personen in Einrichtungen der Kinder- und Jugendhilfe, der Behindertenhilfe, der Sozialpsychiatrie und in Pflegeheimen, denen Besuche weitgehend vorenthalten und für die nahezu sämtliche Freizeit-, Arbeits-, Bildungs- und Therapie-Angebote eingestellt werden,

- Frauen und Kinder, die von häuslicher, durch sozialen Stress induzierter Gewalt bedroht sind,

- Personen, denen Vereinsamung droht.

- Ökonomische Folgen: Die Krise macht, worauf von verschiedener Seite zu Recht hingewiesen wird, nicht nur deutlich, dass in solchen Fällen mehr als lediglich ein handlungsfähiger Staat, nämlich mittel- bis langfristig auch eine funktionierende Marktwirtschaft für die Bewältigung der Situation gebraucht wird. In bestimmten Branchen - etwa der Hotellerie, dem Gastgewerbe, dem Kulturbereich - wird gegenwärtig die wirtschaftliche Existenz vor allem von Kleinunternehmern und Selbstständigen gefährdet, die für ihr tägliches Auskommen auf regelmäßige Einnahmen angewiesen sind. Zugleich verlieren viele Menschen, gerade auch in prekären Arbeitsverhältnissen, ihre Arbeit. Neben absehbaren Wohlstandsverlusten für jedermann durch eine drohende weltweite Rezession sind außerdem Probleme der Versorgung mit Gütern des täglichen Bedarfs und die Sicherung der Kapazitäten und des Know-hows in der Produktion zu bedenken. Nicht zuletzt hängen die unmittelbare Versorgung medizinischer Einrichtungen mit der für die klinische Behandlung notwendigen Ausrüstung und die Sicherung gebotener Hygienestandards von funktionierenden Versorgungsstrukturen ab. Vor allem zu befürchten ist aber ein Zusammenbruch des marktwirtschaftlichen Gesamtsystems, wenn in Deutschland zu viele Unternehmen der mittelständischen Industrie aufgrund naturgemäß meist geringer Kapitalreserven Insolvenz anmelden müssen. Dabei genügt es aus strukturellen Gründen nicht, solche Insolvenzen $\mathrm{zu}$ verhindern; vielmehr sollte es auch darum gehen, das operative $\mathrm{Ge}$ schäft wieder zu ermöglichen. Dies ist nur erreichbar, wenn die komplex vernetzte Interaktion von Produzenten untereinander und mit den Konsumenten im Rahmen der Rechtsordnung wieder hinreichend in Gang kommen kann und ein jedenfalls teilweise normalisiertes Konsumverhalten wieder möglich wird. 
- Elementare Bedingungen demokratischer Kultur: Auf längere Sicht ist es selbst für eine gefestigte Demokratie problematisch, in einem Zustand zu verharren, in dem insbesondere die gerade als Korrektiv und Impulsgeber für die demokratischen Prozesse gedachten Grundrechtsgarantien weitgehend außer Kraft gesetzt sind, oder wenn etwa Wahlen verschoben werden oder auf Briefwahl gesetzt wird. Für den Rechtsstaat ist es zudem elementar wichtig, nicht in ein Denken in Kategorien des Ausnahmezustands zu verfallen.

Diese Überlegungen zu den Nebenfolgen des Lockdowns müssen ergänzt werden durch Kriterien, die politische Entscheidungen über die Fortsetzung, Lockerung oder Beendigung der Strategie der sozialen Distanzierung anleiten können. Dafür lassen sich zunächst drei Konstellationen unterscheiden:

I Die Strategie ist insoweit erfolgreich, als eine Überlastung des Gesundheitssystems vermieden werden kann und andere gesundheitliche, wirtschaftliche und politische Schäden nicht überwiegen. Eine solche Situation ist erreicht, wenn die Zahl der Menschen, die eine infektiöse Person ansteckt, statistisch betrachtet dauerhaft unter eins liegt. Wenn und soweit dieser Zustand erreicht wird, ist der schrittweise und epidemiologisch evaluierte Abbau der Restriktionen nicht nur möglich, sondern geboten.

II Die Strategie führt innerhalb eines gesetzten Zeitraums - dessen Länge zu bemessen wäre nach der unsicheren epidemiologischen Prognose, wann die ergriffenen Maßnahmen Wirkung zeigen sollten nicht zu dem gewünschten Erfolg der Vermeidung einer Überlastung des Gesundheitssystems, oder es überwiegen andere gesundheitliche, wirtschaftliche und psychosoziale Schäden. In dieser Situation endet die Legitimität der Strategie.

III Es gibt die begründete Hoffnung, dass die Fortsetzung der Strategie über einen definierten Zeitraum dazu führt, dass der bereits eingetretene Zustand der Überlastung des Gesundheitssystems revidiert wird. Auch in einer solchen Situation sind zumindest Lockerungen des Restriktionsregimes angezeigt. Kollidierende Interessen gewinnen zunehmend an Gewicht.

Soweit man diese Interessen überhaupt für einen bestimmten Zeitraum (Konstellation I) als nachrangig einzustufen bereit ist, dürften sie sich jetzt als starke Gründe für die Durchbrechung der Strategie erweisen. Entsprechende Überlegungen gelten für die Berücksichtigung ökonomischer Folgeerwägungen. Die absehbare weltweite Rezession, der massive Rückgang des Bruttoinlandprodukts und die damit verbundenen Belastungen der öffentlichen Haushalte lassen sich nämlich mit quantitativen Kennziffern nicht hinreichend erfassen. Sie haben, wie die skizzenhafte Zusammenstellung deutlich gemacht hat, Auswirkungen auf existenzielle Funktionsbedingungen eines Gemeinwesens, dessen sozialstaatliche Solidarität auf wirtschaftliche Leistungsfähigkeit angewiesen ist.

Solche und ähnliche Überlegungen bedürfen der ernsthaften gesellschaftlichen Debatte auch schon in Zeiten der Krise. Dabei wird auch zu erörtern sein, welche Lebensrisiken eine Gesellschaft als akzeptabel einzustufen gewillt ist und welche nicht. Die in den kommenden Monaten erforderliche Bestandsaufnahme und Evaluation wird dabei auch den rechtlichen Ordnungsrahmen eingehend $\mathrm{zu}$ analysieren haben - nicht zuletzt mit kritischem Blick auf die neuetablierten Eingriffsbefugnisse.

\section{c) Mögliche Elemente des Vorgehens}

Wesentlicher Orientierungspunkt für das Vorgehen in der kommenden Zeit ist die weitgehende Vermeidung der be- schriebenen Triage-Situationen. Zugleich sollten ergriffene Maßnahmen in einem dynamischen Prozess regelmäßig re-evaluiert werden, um Belastungen und Folgeschäden so gering wie möglich zu halten. Und schließlich sollten parallel Anstrengungen unternommen werden, langfristige Lösungen möglichst bald verfügbar zu machen. Im Folgenden wird daher, ohne eine Priorisierung andeuten zu wollen, eine Reihe von Vorgehensweisen und Maßnahmen empfohlen, die in der nächsten Zeit im Vordergrund stehen sollten:

- Stärkung und Stabilisierung der Kapazitäten des Gesundheitswesens insbesondere der Pflege, Einführung eines flächendeckenden Systems zur Erfassung und optimierten Nutzung von Intensivkapazitäten.

- Bessere Vernetzung der Akteure im Gesundheitssystem und mit anderen relevanten Gesellschaftsbereichen.

- Weiterer Ausbau der Testkapazitäten zur Diagnostik und zum Nachweis von (vorläufiger) Immunität (serologisch, derzeit in Entwicklung).

- Weitere kontinuierliche Datensammlung zu individueller und Gruppenimmunität, (Gemeinschaftsschutz), zu Verläufen von Covid-19 etc., weitere Modellentwicklung, um Effektivität von Interventionen abschätzen zu können.

- Breite Förderung/Unterstützung von Forschung zu Impfstoffen und Therapeutika sowie Vorbereitung von Förderstrukturen für deren massenhafte Produktion und Einführung.

- Unterstützung von interdisziplinärer Forschung unter anderem zu sozialen und psychischen Folgen der Covid-19-Pandemie, einschließlich zukünftiger Risikowahrnehmungen.

- Kontinuierliche Re-Evaluation von freiheitsbeschränkenden Maßnahmen; wo vertretbar, deren schrittweise Zurücknahme und eine Wiederaufnahme sozialer und ökonomischer Aktivität.

- Entwicklung von effektiven und erträglichen Schutz-/ Isolationsstrategien für Risikogruppen (Vorerkrankte, ältere Menschen) und in bestimmten Institutionen (etwa Einrichtungen der Alten- und Langzeitpflege).

- Insbesondere für jüngere Hochrisikogruppen ist zu beachten, dass eine wirksame Selbstisolation die Möglichkeit zur (präventiven) Krankschreibung oder zur sonstigen Freistellung der jeweils Betroffenen und ihrer Haushaltsangehörigen zwingend voraussetzt.

- Fundierte Informationsstrategie: Transparente und regelmäßige Kommunikation über die ergriffenen Maßnahmen und zur politischen Entscheidungsfindung im Zusammenhang mit hochinfektiösen Erkrankungen.

- Konkrete Berechnungen der zu erwartenden Kosten durch ergriffene Maßnahmen und Alternativszenarien.

Krisen, so heißt es oft, seien die „Stunde der Exekutive". Das greift zu kurz. Gerade in der Krise ist auf das Zusammenwirken des gewaltengegliederten und zudem föderal differenzierten Staates mit der Vielfalt gesellschaftlicher und namentlich wissenschaftlicher Stimmen zu setzen. Die aktuell zu klärenden Fragen berühren die gesamte Gesellschaft; sie dürfen nicht an einzelne Personen oder Institutionen delegiert werden. Gerade schmerzhafte Entscheidungen müssen von den Organen getroffen werden, die hierfür durch das Volk mandatiert sind und dementsprechend auch in politischer Verantwortung stehen. Die Corona-Krise ist die Stunde der demokratisch legitimierten Politik.

Die Akademie für Ethik in der Medizin pflegt eine laufend aktualisierte Liste mit „Empfehlungen und Materialien zu ethischen Fragen der Patientenversorgung angesichts der COVID-19-Pandemie": https://www.aem-online.de/ index.php? id =163 [26.3.2020]. 


\title{
Mitglieder des Deutschen Ethikrates
}

(zum Zeitpunkt der Veröffentlichung dieser Empfehlung)

Prof. Dr. theol. Peter Dabrock (Vorsitzender)

Prof. Dr. med. Katrin Amunts

(Stellvertretende Vorsitzende)

Prof. Dr. iur. Dr. h. c. Volker Lipp

(Stellvertretender Vorsitzender)

Prof. Dr. med. Claudia Wiesemann

(Stellvertretende Vorsitzende)

Constanze Angerer

Prof. Dr. iur. Steffen Augsberg

Prof. Dr. theol. Franz-Josef Bormann

Prof. Dr. med. Alena M. Buyx

Prof. em. Dr. iur. Dr. h.c. Dagmar Coester-Waltjen

Dr. med. Christiane Fischer

Prof. em. Dr. phil. habil. Dr. phil. h.c. lic. phil.

Carl Friedrich Gethmann
Prof. Dr. theol. Elisabeth Gräb-Schmidt

Prof. Dr. rer. nat. Dr. phil. Sigrid Graumann

Prof. Dr. med. Wolfram Henn

Prof. Dr. iur. Wolfram Höfling

Prof. Dr. (TR) Dr. phil. et med. habil. Ilhan Ilkilic

Prof. Dr. rer. nat. Ursula Klingmüller

Stephan Kruip

Prof. Dr. phil. Dr. h.c. Dipl.-Psych. Andreas Kruse

Prof. Dr. phil. Adelheid Kuhlmey

Prof. Dr. med. Leo Latasch

Prof. Dr. theol. Andreas Lob-Hüdepohl

Prof. em. Dr. iur. Reinhard Merkel

Prof. Dr. phil. Judith Simon

Prof. Dr. med. Elisabeth Steinhagen-Thiessen

Dr. phil. Petra Thorn

\section{Die wirtschaftliche Unabhängigkeit der ärztlichen Fortbildung}

\author{
Quadratur des Kreises oder realitätsfernes Wunschdenken?
}

\section{Albrecht Wienke und Lisa Hübner}

\begin{abstract}
Ärzte ${ }^{\star}$ in Deutschland sind gesetzlich ${ }^{1}$ zur berufsbegleitenden Fortbildung verpflichtet. Angebote hierzu gibt es mehr als genug; offenbar ist die ärztliche Fortbildungspflicht für den einen oder anderen Anbieter solcher Fortbildungsveranstaltungen ein wirtschaftlicher Selbstläufer. Doch seit geraumer Zeit herrscht eine rege Diskussion zur Legitimation der Finanzierung und Zertifizierung dieser Fortbildungsveranstaltungen ${ }^{2}$. Denn eine Vielzahl von Fortbildungen wird durch Unternehmen aus der Gesundheitsbranche finanziell und personell unterstützt. Dies gilt in besonderem Maße für die Kongresse und Jahresversammlungen der wissenschaftlichen medizinischen Fachgesellschaften. Dort treffen sich Jung und Alt der jeweiligen Fachgebiete, um Erfahrungen und Neuigkeiten der jeweiligen medizinischen Fachrichtung darzustellen und untereinander auszutauschen. Auch die Angebote für ärztliche Fortbildungen in Kursen oder anderen didaktisch wirksamen Verfahren nehmen erkennbar zu. Wesentliche Triebfeder solcher Veranstaltungen ist die Pharma- und Medizinprodukteindustrie, die solche Jahresversammlungen nutzt, um ihrerseits den pharmakologischen und medizin-technischen Fortschritt zu präsentieren. Nicht umsonst sprechen Insider in diesem Zusammenhang nicht von Messen oder Ausstellungen der Pharma- und Medizinprodukteunternehmen, sondern von einer (wissenschaftlich getriggerten) Präsentation der Fachindustrie. Dies soll von dem Vorwurf einer allein von den Unternehmen der Gesundheitsbranche
\end{abstract}

Rechtsanwalt Dr. iur. Albrecht Wienke,

Fachanwalt für Medizinrecht,

Rechtsanwältin Lisa Hübner,

Wienke \& Becker - Köln, Deutschland wirtschaftlich unterstützten und beeinflussten Tagung ablenken. Einige Stimmen bangen vor diesem Hintergrund um die ärztliche Unabhängigkeit und verlangen eine strikte Trennung von Ärzteschaft und Industrie im Zusammenhang mit ärztlichen Fortbildungen. Gewünscht und anerkannt werden ausschließlich Fortbildungsveranstaltungen ohne Beteiligung von Industrieunternehmen der Gesundheitsbranche. Um gesponserten Fortbildungen die Attraktivität $\mathrm{zu}$ entziehen, wird gefordert, die Teilnahme an solchen Veranstaltungen nicht mehr mit CME-Punkten zu zertifizieren ${ }^{3}$.

Die Realität spricht indes eine andere Sprache:

Fortbildungsveranstaltungen und medizinisch wissenschaftliche Großveranstaltungen der wissenschaftlichen medizinischen Fachgesellschaften mit bis zu 10.000 Teilnehmern sind ohne eine massive wirtschaftliche Beteiligung der Pharma- und Medizinprodukteindustrie nicht mehr denkbar. Dabei sind die von den ärztlichen Teilnehmern verlangten Gebühren vergleichsweise überschaubar. In anderen Branchen müssen die Teilnehmer von Fortbildungsveranstaltungen tief in die (eigene) Tasche greifen, um sich im je-

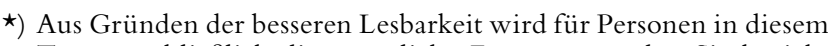
Text ausschließlich die männliche Form verwendet. Sie bezieht sich auf Personen aller Geschlechter $(\mathrm{m} / \mathrm{w} / \mathrm{d})$.

1) $\$ 95 \mathrm{~d}$ Abs. 1 SGB V, $\$ 4$ Abs. $1 \mathrm{MBO}-\ddot{\mathrm{A}}$.

2) Dazu und zu der gesamten Problematik ausführlich Balzer, Die Akkreditierung industrieunterstützter Fortbildungsveranstaltungen nach Umsetzung des GKV-Modernisierungsgesetzes - eine Reform der Reform?, MedR 2004, 76, 76 ff.

3) So die konkrete Forderung von Lempert/Schott/u. a.: Fortbildungen, Keine Punkte bei Sponsoring, DÄBl. 13/2019, S. A620, S. A620 ff. 